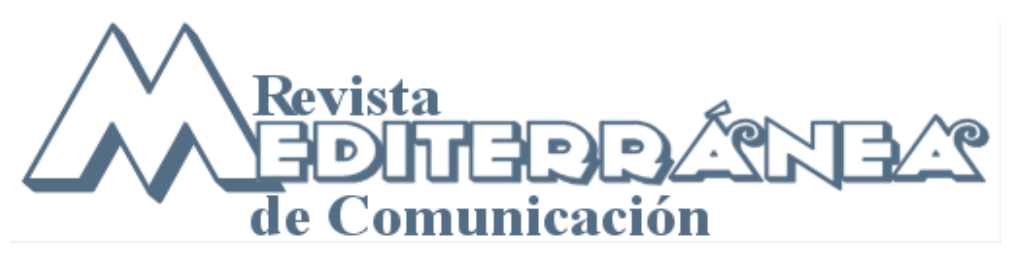

Año 2 (2011), pp. 43-58 ISSN 1989-872X

\title{
Integración de las tecnologías de la información y la comunicación (TIC) en la universidad: diez propuestas de aprendizaje
}

\section{Integration of information technology and communication technology (ICT) in university teaching: ten proposals for learning}

\author{
Fátima Martínez Gutiérrez \\ Universidad San Pablo CEU, USP-CEU \\ fatima.martinezgutierrez@ceu.es \\ Yanet Acosta \\ Universidad Camilo José Cela, UCJC \\ myacosta@ucjc.edu
}

\section{Resumen}

Este artículo pretende resaltar la enorme importancia que pueden adquirir la integración de las Tecnologías de la Información y de la Comunicación (TIC) si se realiza un nuevo planteamiento docente en las aulas universitarias. Los profesores han de embarcarse en nuevos conocimientos informáticos y los alumnos han de aprender a adaptar espacios virtuales destinados para el ocio y el entretenimiento, como Facebook, Tuenti o MySpace, en escenarios de aprendizaje y formación, favoreciéndoles al mismo tiempo una mayor interactividad con el profesor. Al mismo tiempo que se reflexiona sobre la nueva docencia universitaria con la ayuda de herramientas 2.0, reseñaremos 10 plataformas online, gratuitas y de fácil manejo, para impulsar la información entre los alumnos, basado en la experiencia de las autoras con el desarrollo de esta nueva metodología en línea. Este nuevo modelo pedagógico se apoyaría en el concepto entendido como blendedlearning, es decir, en la manera de compatibilizar clases magistrales con aquellas clases trasladadas a la esfera digital. En consecuencia, a la reflexión teórica se añaden ejemplos concretos como Facebook, Google groups, Twitter o Ning. Advertimos que este artículo no presenta resultados concretos puesto que su pretensión es la de elaborar una propuesta de innovación docente que pueda ser aplicable a la hora de impartir clases en la universidad.

Palabras claves

Educación; Comunicación; Enseñanza-Aprendizaje; Internet.

\section{Abstract}

This article aims to highlight the enormous importance that the integration of Information Technology and Communication (ICT) can get if it takes a new approach to teaching in university classrooms. Teachers need to embark in new computer skills and the students have to learn to 
adapt virtual spaces for leisure and entertainment such as Facebook, MySpace or Tuenti in learning and training scenarios, while promoting greater interactivity teacher. While reflecting on the new university teaching with the help of tools 2.0 , reviews 10 platforms online, free and easy to use, to increase information among students, based on the experience of the authors with the development of this new online methodology. This new educational model will build on the concept understood as blended-learning, in other words, how to reconcile those classes with classes moved to the digital realm. Consequently, the theoretical specific examples are added as Facebook, Google groups, Twitter or Ning. We note that this article does not show concrete results since its aim is to draw up a proposal for teaching innovation that may apply when teaching at the university.

\section{Key Words}

Education; Communication; Teaching and Learning; Internet.

Sumario: 1. Introducción. 2. El aula digital: del e-learning a las redes sociales. 2.1. Combinar formación off-line y on-line. 2.2. La filosofía Web 2.0. 2.3. La posuniversidad y las redes sociales. 3. Nuevos métodos de aprendizaje: herramientas en la Red. 3.1. Los blogs. 3.2. Google: documentos y grupos. 3.3. Posterous. 3.4. Tuenti. 3.5. Facebook. 3.6. Twitter. 3.7. Crear una red propia: Ning. 3.8. Rastrear el seguimiento de los alumnos: Bit.ly. 3.9. Encuestas online. 3.10. Tutorías a través de aplicaciones de móvil: Whatsapp. 4. Conclusiones. 5. Bibliografía.

Summary: 1. Introduction. 2. The digital classroom: e-learning in social networks. 2.1. Combining off-line training and on-line. 2.2. Web 2.0 philosophy. 2.3. The university and social networks. 3. New methods of learning: tools in the Web 3.1. Blogs. 3.2. Google: documents and groups. 3.3. Posterous. 3.4. Tuenti. 3.5. Facebook. 3.6. Twitter. 3.7. Create your own network: None. 3.8. Track monitoring students: Bit.ly. 3.9. Online surveys. 3.10. Tutoring through mobile applications: WhatsApp. 4. Conclusions. 5. Bibliography.

\section{Introducción}

Las Tecnologías de las Información y de la Comunicación, en adelante TIC, constituyen un medio de acceso instantáneo a la información que jamás había existido, hoy más que nunca, la proliferación de las nuevas tecnologías parecen estar dando al traste con los ya obsoletos paradigmas comunicativos, que han sido instaurados durante siglos. Por ende, la educación universitaria actual se encuentra en una coyuntura de cambio, la aceleración de las nuevas tecnologías en la sociedad están provocando numerosas transformaciones en el aprendizaje de los más jóvenes. Este cambio de paradigma está vinculado al poder del usuario, convertido ahora en webactor (Pisani y Piotet, 2008), que está influyendo de manera significativa en los nuevos métodos de enseñanza-aprendizaje virtual. En otras palabras, urge una alfabetización digital entre todas las partes implicadas, centros educativos, profesores o docentes y alumnos o estudiantes, una alfabetización que requerirá, entre otros aspectos, una necesaria combinación entre las clases magistrales y las clases que alcanzan entornos más allá del espacio físico del aula, como sucede en el ciberespacio. Es la idea anglosajona conocida como blended-learning, que promueve el aprendizaje a distancia con las clases en el aula. 
En 1994, Lévy acuñaría el concepto de inteligencia colectiva y abordaría un asunto, que por aquel entonces, se perfilaba en un horizonte muy lejano, cuando intentaba explicar el término de inteligencia colectiva y profundizar en la antropología del ciberespacio, un nuevo contexto al que ha de adaptarse el profesorado, muchas veces desorientado ante estudiantes, cada vez más involucrados en los nuevos dispositivos móviles y cuyas dinámicas de aprendizaje están ligadas a una alfabetización digital que se ha incrementado exponencialmente en menos de una década. Parafraseando a Buckingham, no parece andar desencaminado el argumento que afirma que, por lo que se refiere a la educación mediática, los profesores han dejado de ser los expertos (Buckingham: 2005: 120). Se puede hablar de un desfase, de una disonancia cognitiva entre profesores y alumnos, puesto que da la impresión de que muchos alumnos han tomado la delantera en este terreno frente a una gran mayoría del profesorado, en muchas ocasiones ajenos a las tecnologías de última generación.

¿Cuál es el dominio de los alumnos frente a las herramientas ofrecidas por el ordenador o el móvil conectado a Internet, pantallas frente a las cuales dedican varias horas a la semana? Es una pregunta que deberían hacerse los docentes que deseen implementar nuevas fórmulas pedagógicas en las aulas. El profesor F. García se lo plantea de la siguiente manera: "¿cuáles son realmente las características que lo definen?, ¿son un grupo homogéneo?, ¿disponen de competencias, habilidades y conductas similares?, ¿cuál es la frontera real que separa a los nativos y a los inmigrantes digitales?" (García, 2009: 7).

Se está empezando a dar por supuesto que los profesores universitarios se encuentran con nativos digitales (Digital Natives), como cercioraba Prensky, o por la Generación Red (Net Generation), en palabras de Tapscott, pero lo cierto es que desconocemos en gran medida los diferentes usos concedidos a las nuevas tecnologías, aunque parezca temprano analizar los usos y abusos de un sector de la población juvenil, cada vez mayor, volcada en la conexión continua a Internet. La causa, el progreso fulminante de la técnica de la comunicación: la telefonía (1880), la radio (1900), la televisión (1930), la informática (1940) y las redes (1980) (Wolton, 2010: 27). Si el teléfono tardó 70 años en convertirse en un medio de comunicación popular, la radio precisó de 30, la televisión de 15 y la prensa en Internet sólo de cuatro o cinco (Parra y Álvarez, 2004: 59).

Según un estudio norteamericano, en 2009, las horas de conexión a la Red aumentaban hasta diecisiete horas semanales en los adultos, mientras que para los adultos más jóvenes, los veinteañeros superaban las diecinueve horas a la semana online (Carr, 2011: 99). En palabras del filósofo francés D. Wolton, "el tiempo, principal enemigo de Internet" (Wolton, 2010: 54), en palabras del experto en ciberperiodismo, José Cervera, la economía de la atención, clave en la infoxicación diseminada en la Red. En febrero de 2009 se publicaba un artículo titulado Well connected? The biological implications of "social networking" que alertaba sobre el número de horas dedicadas diariamente al uso de aparatos electrónicos, duplicado por dos desde 1987, en detrimento de la interacción cara a cara, que pasaba de seis a dos horas diarias (Redondo, 2010: 18).

Se pone de manifiesto que los nuevos medios de comunicación, lejos de ser aburridos, pueden integrarse en las nuevas pedagogías de educación superior. Nuestra tesis defenderá un nuevo método de aprendizaje mediante el uso de todas las herramientas facilitadas por Internet, como suceden con el acentuado uso de los blogs y con las adictivas redes sociales, que bien pudieran servir de plataformas de trabajo para materias impartidas en las facultades de Comunicación, extensible a facultades de otras áreas de conocimiento aplicadas. El verdadero reto de la educación, asegura el profesor Tejedor, no está en la innovación tecnológica sino en la innovación pedagógica, que debería incluir el uso de las herramientas didácticas más apropiadas (entre ellas 
las TIC), para diseñar actividades de aprendizaje de calidad para los estudiantes, bajo los parámetros de modelos educativos constructivistas y colaborativos (Sierra y Sotelo, 2010: 499).

Internet no sólo es un medio de difusión personal, sino que implica un importante movimiento comercial, unas veces remunerado y, otras muchas, completamente gratuitas. Es el llamado modelo crowdsourcing, que consiste en invitar a "la multitud" de los usuarios, a través de la web, a realizar tareas que antes se efectuaban dentro de una organización; a cambio del mínimo costo, o incluso gratis (Piotet y Pisan; 2010: 197). Por lo tanto, millones de personas la utilizan para distribuir sus propias creaciones digitales, en forma de blogs, fotos, canciones y podcats, así como para criticar, editar o modificar las creaciones de los demás. La vasta enciclopedia escrita por voluntarios en Wikipedia, el servicio de vídeo de Youtube, producido en gran parte por aficionados, el enorme repositorio de fotos Flickr, el extenso compendio de blogs que es Huffington Post... (Carr; 2011: 109).

De esta manera, se está llevando a la práctica la idea promulgada por el creador de la world wide web, Tim Berners-Lee, sobre la intercreatividad, cuyo fin no era otro que el "de hacer cosas o el de poder resolver problemas juntos"; término que aúna en sí otros dos; el de interactividad y el de creatividad. El teléfono móvil, por ejemplo, fascina a los nativos digitales, y ha seducido a quienes no lo son, porque en él convergen juegos, vídeos, fotos, música, textos y la posibilidad de mantener una comunicación ubicua con sus contactos vía sms, mms y llamadas (Prensky, 2010).

\section{El aula digital: del e-learning a las redes sociales}

Lawrence Lessig nos enseñó que el ciberespacio no es un lugar sino muchos lugares. Estos lugares no vienen dados, sino que se construyen, al menos parcialmente, por las arquitecturas que configuran diferentes espacios y promueven ciertas prácticas - lo que las personas pueden o no hacer - de acuerdo con determinados principios (Piscitelli, 2010: 71). El aula digital, fruto del trabajo de un alumno frente a la pantalla de un ordenador no tiene por qué constituir una novedad en nuestros días, de hecho, lleva desarrollándose durante varios años, a modo de másters on line o de cursos especializados en línea, que no requieren de la asistencia del alumno a un espacio común determinado. Esta pedagogía es entendida como métodos de formación e-learning.

Los modelos de enseñanza están evolucionando hacia metodologías adaptadas a nuevos entornos. Una perspectiva de vanguardia de las teorías pedagógicas que implica a diversos protagonistas. De entre ellos destacan tres, a saber: los estudiantes como motor de cambio, los docentes en su nuevo papel de mediadores y facilitadores de estructuras más que de conceptos y el entorno empresarial que fuerza al académico a hacer un esfuerzo por adaptar los conocimientos a la práctica real (Sierra y Sotelo; 2010: 549). La universidad ha de apostar por la innovación digital y los nuevos modelos de enseñanza - aprendizaje, dispuestos por las nuevas tecnologías, incluso con cursos de formación para los docentes; el profesor, con formación suficiente para seguir este proceso de adaptación, sabrá sacar el máximo rendimiento a sus estudiantes (Sierra y Sotelo; 2010: 568).

\subsection{Combinar formación off-line y on-line}

No es de extrañar que los docentes, procedentes de ámbitos académicos-universitarios, se estén planteando seriamente la manera de compatibilizar las clases presenciales en las aulas con las prácticas digitales mediante el uso de Internet. Una teoría que está siendo respaldada por académicos, conscientes de las transcendentales repercusiones de los espacios web 2.0. En consecuencia, aseguran profesores de la Universidad de Salamanca, el sistema combinado entre 
las clases magistrales y el e-learning facilitará a los alumnos no sólo aprender conceptos nuevos sino también a afianzarlos, aumentando así la autonomía y la motivación de los estudiantes por diferentes temas, puesto que van a ser partícipes de ellos (Sierra y Sotelo; 2010: 596). Algunas de las ventajas subrayadas por estos autores son las siguientes:

- Inmersión práctica en un entorno Web 2.0

- Eliminación de las barreras espaciales y temporales (desde su propia casa, en el trabajo, en un viaje a través de dispositivos móviles, etc.)

- Prácticas en entornos de simulación virtual, difíciles de conseguir en formación presencial, sin una gran inversión.

- Gestión real del conocimiento: intercambio de ideas, opiniones, prácticas, experiencias. Enriquecimiento colectivo del proceso de aprendizaje sin límites geográficos.

- Actualización constante de los contenidos.

- Reducción de costes.

- Permite una mayor comunicación y feedback entre alumnos y profesores.

Por un lado, se fomenta la creatividad en el trabajo del alumnado, y por otro, el profesor puede servir de guía y puede fomentar su comunicación con ellos. Como indica Buckingham, el potencial participativo de las nuevas tecnologías - especialmente de Internet - ha facilitado significativamente, por una parte, la participación de los jóvenes en la producción mediática creativa y, por otra parte, las iniciativas que en este mismo sentido puedan tomar profesores con sus alumnos (Buckingham, 2005: 36). En este sentido, no sólo se incentiva la creatividad del alumno, sino también se impulsa la creatividad en las nuevas formas pedagógicas del docente en cuestión, al tener que plantear nuevos ejercicios, trasladados a la esfera digital.

\subsection{La filosofía Web 2.0}

El término Web 2.0 se debe a Darcy DiNucci que lo citó en 1999 en uno de sus artículos en referencia al cambio que estaba sufriendo la web por entonces, aunque empezó a popularizarse a partir del año 2004, cuando O'Reilly Media y MediaLive organizaron la primera conferencia sobre Web 2.0 (De Haro; 2010: 24). A continuación presentamos una tabla entre las diferencias entre Web 1.0 y Web 2.0, elaborada por el profesor J. J. de Haro, creador de Eduredes, una red social destinada para los profesores:

\begin{tabular}{|l|l|}
\hline \multicolumn{1}{|c|}{ Web 1.0 } & \multicolumn{1}{c|}{ Web 2.0 } \\
\hline Web para leer & Web para leer y escribir \\
\hline Era de los editores locales HTML & Era de la edición en línea \\
\hline $\begin{array}{l}\text { Trabajo con aplicaciones instaladas en el } \\
\text { ordenador personal }\end{array}$ & $\begin{array}{l}\text { Trabajo a través de aplicaciones que se } \\
\text { encuentran en la red (la nube) }\end{array}$ \\
\hline $\begin{array}{l}\text { El sistema operativo es lo más importante } \\
\text { del ordenador }\end{array}$ & $\begin{array}{l}\text { El sistema operativo pierde importancia el } \\
\text { navegador de Internet se convierte en la } \\
\text { pieza fundamental del ordenador }\end{array}$ \\
\hline $\begin{array}{l}\text { Las empresas monopolizan toda la red, lo } \\
\text { importante es vender }\end{array}$ & $\begin{array}{l}\text { Los usuarios crean relaciones entre ellos, } \\
\text { lo importante son las redes de personas }\end{array}$ \\
\hline $\begin{array}{l}\text { Los usuarios se ocultan tras los apodos } \\
\text { (nicks) }\end{array}$ & $\begin{array}{l}\text { Los usuarios se identifican con su } \\
\text { nombre real y su foto }\end{array}$ \\
\hline
\end{tabular}

Figura 1: Elaborado por Juan José de Haro, Redes sociales para la educación. 
Castells se refiere a la capacidad de las nuevas tecnologías de esta manera: "Lo que caracteriza a la revolución tecnológica actual no es el carácter central del conocimiento y la información, sino la aplicación de ese conocimiento e información a aparatos de generación de conocimiento $y$ procesamiento de la información / comunicación, en un círculo de retroalimentación acumulativo entre la innovación y sus usos" (Castell, 1999: 47).

\subsection{La posuniversidad y las redes sociales}

Un ejemplo significativo llevado a cabo en Argentina es el trabajo denominado El proyecto Facebook y la posuniversidad. Sistemas operativos sociales y entornos abiertos de aprendizaje, donde se ha intentado poner en práctica la docencia universitaria en la red social de Facebook. En esencia, este proyecto, fue un intento de construcción de un entorno colaborativo y abierto de educación, que se ajustará más a las maneras en que entendemos que se produce el conocimiento y menos a una tradición educativa que concibe a los alumnos como destinatarios y no actores de este proceso (Piscitelli et alli, 2010: 15). Piscitelli explica que Facebook "no debe ser concebido como una herramienta de educación", sino como "una herramienta en la cual aprender". Los profesores argentinos, responsables del Proyecto Facebook, han recogido el término acuñado por Jim Groom (Downes, 2008), edupunk, cuyo papel debe ser que cada uno cree su propio proceso de aprendizaje, utilizando los materiales que estén al alcance de su mano, para avanzar en la agenda propia y no en una lógica y un lenguaje de participación entregados intactos a la sociedad (Piscitelli et al., 2010: 12).

En otras palabras, El proyecto Facebook concibe la idea de la posuniversidad, a universidad nacida de la exploración de alternativas digitales, que se extienden más allá de los modelos de transmisión tradicional, donde los instructores (y los libros) difunden y donde los estudiantes escuchan pasivamente (Piscitelli et al., 2010: 13). En esta línea también han trabajado profesores de universidades españolas como Linda Castañeda, reflejado en la obra coordinada por esta profesora de la universidad de Murcia, Aprendizaje con redes sociales. Tejidos educativos para los nuevos entornos. En este estudio se distingue tres tipos de herramientas en Internet (Castañeda, 2010: $20-25)$ :

- Los medios de comunicación social (Social Media): herramientas más tradicionales de la Web 2.0; aquellas que tienen como objetivo que sus usuarios publiquen y compartan un determinado elemento de comunicación concreto y que se estructuran a esos elementos.

- Los medios de seguimiento de la actividad en Red (Lifestreaming): herramientas de sindicación de contenidos (casi siempre a través de RSS), que recogen la actividad generada por una persona en la red en todos sus sitios web.

- Las redes sociales (Social Networking Sites): herramientas telemáticas que permiten a un usuario crear un perfil de datos sobre sí mismo en la red y compartirlo con otros usuarios. Según la teoría de grafos, una red social es una estructura social que se puede representar mediante nodos conectados por aristas. Los nodos representan los individuos y las aristas, las relaciones entre ellos (De Haro; 2010: 38).

\section{Nuevos métodos de aprendizaje: herramientas en la Red}

El método entendido como el camino por el cual guiaremos nuestro trabajo. En este caso el objetivo es claro: el aprendizaje mediante sistemas informáticos de carácter colaborativo y participativo, rasgos propios de las siguientes herramientas 2.0. En el plano educativo se ha dado en llamar la Acción Colaborativa en Educación (ACE), basada principalmente en la investigación 
de nuevos modelos educativos con el objetivo de mejorar la práctica docente. Cliff et al. (1991) definen la acción de colaborar como la de trabajar juntos dos o más personas para conseguir una meta.

\subsection{Los blogs}

Generar un sitio personal web, de actualización continua, cómodo de manejar y completamente gratuito en forma de bitácora personal nunca fue tan fácil como hasta ahora. El potencial creativo de los blogs ha supuesto toda una revolución en Internet, que bien utilizado puede dar un enorme juego entre profesores y alumnos.

Desde crear un blog relacionado a un materia determinada hasta incentivar la creación de diferentes blogs a todo los alumnados con el fin de adentrarles en la era digital y de hacerles partícipes de publicar entradas, ya sea a modo de comentarios escritos, ya sea con la inclusión de imágenes, vídeos y enlaces. El blog es una herramienta idónea en este nuevo proceso de formación y de transmisión de conocimientos. El estudiante explora, investiga y publica de primera mano contenidos que hasta entonces han estado reservados para doctos en la asignatura.

\subsection{Google: documentos y grupos}

Google es mucho más que un simple buscador en Internet, la enorme cantidad de servicios ofertados, la convierten una de las páginas con más posibilidades de colaboración 2.0, que abarca desde Google News hasta Google Maps. Las aplicaciones que Google, por tanto, pone en manos de los profesionales una infinidad de posibilidades a los profesionales en el ámbito educativo, no sólo por sus herramientas de búsqueda como Google Books y Google Academics, sino también por sus dispositivos de almacenamiento y manejo de documentos, como es Google Docs. Además, ofrece al profesorado la posibilidad de crear un grupo con los mails de sus alumnos, de manera que facilita la gestión del envío de correos electrónicos así como la consulta de los enviados.

\subsubsection{Google groups}

Permite enviar mails conjuntos con toda facilidad y consultar en una página principal, cerrada, a los miembros ordenadamente los mails remitidos, al mismo tiempo que registra las conversaciones mantenidas con cada uno de ellos. 


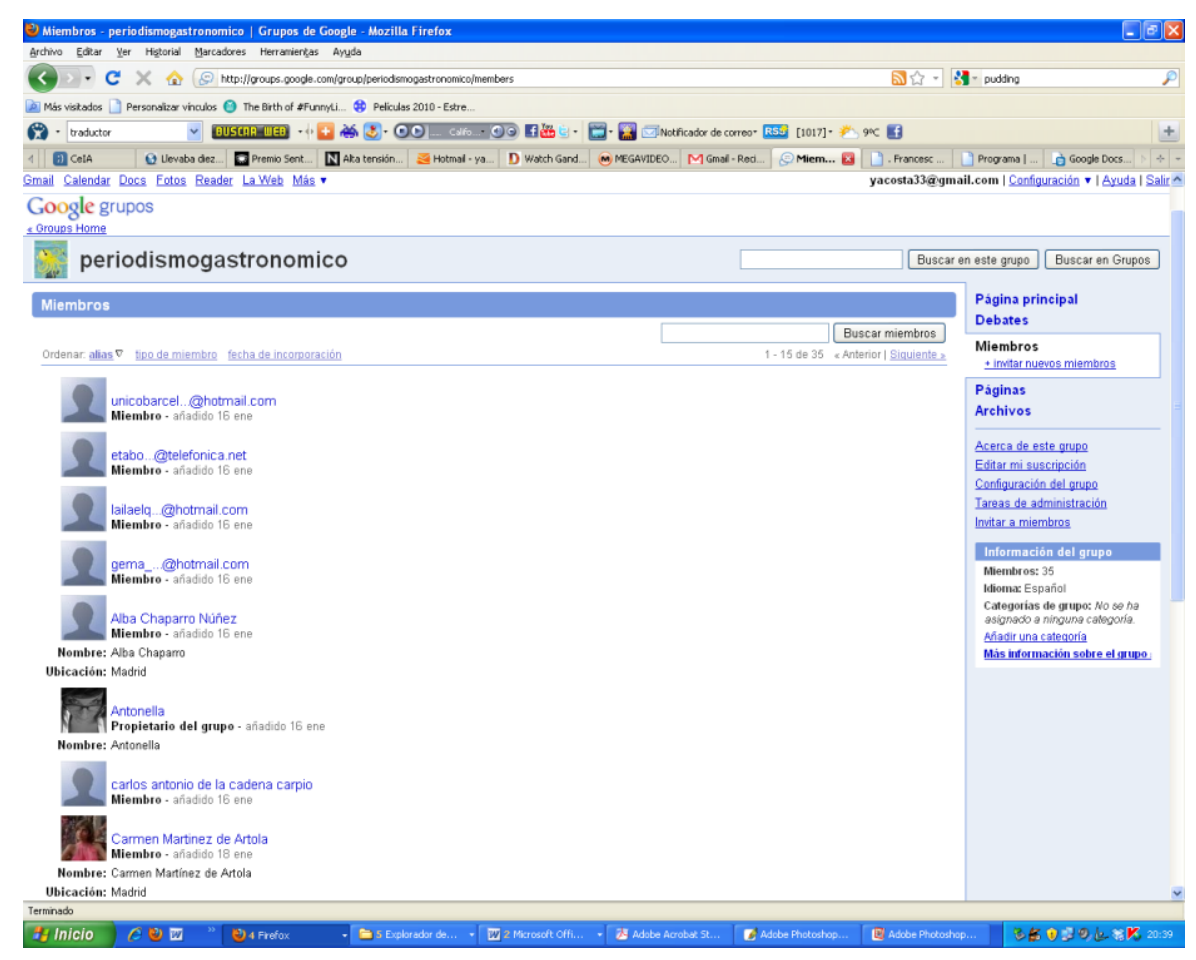

Figura 2: Imagen de Google groups

Los alumnos pertenecientes a este grupo, así como los profesores, se pueden identificar mediante el uso de fotos. En los grupos de Google aparecen registrados todos los mails remitidos desde un mail específico del grupo, el que lo ha generado. Además, puede guardar conversaciones o debates mantenidas online, de forma que facilita la búsqueda de un tema concreto planteado meses antes o de un mail en el que se solicitaba a todos los alumnos la elaboración de un trabajo, etc. 


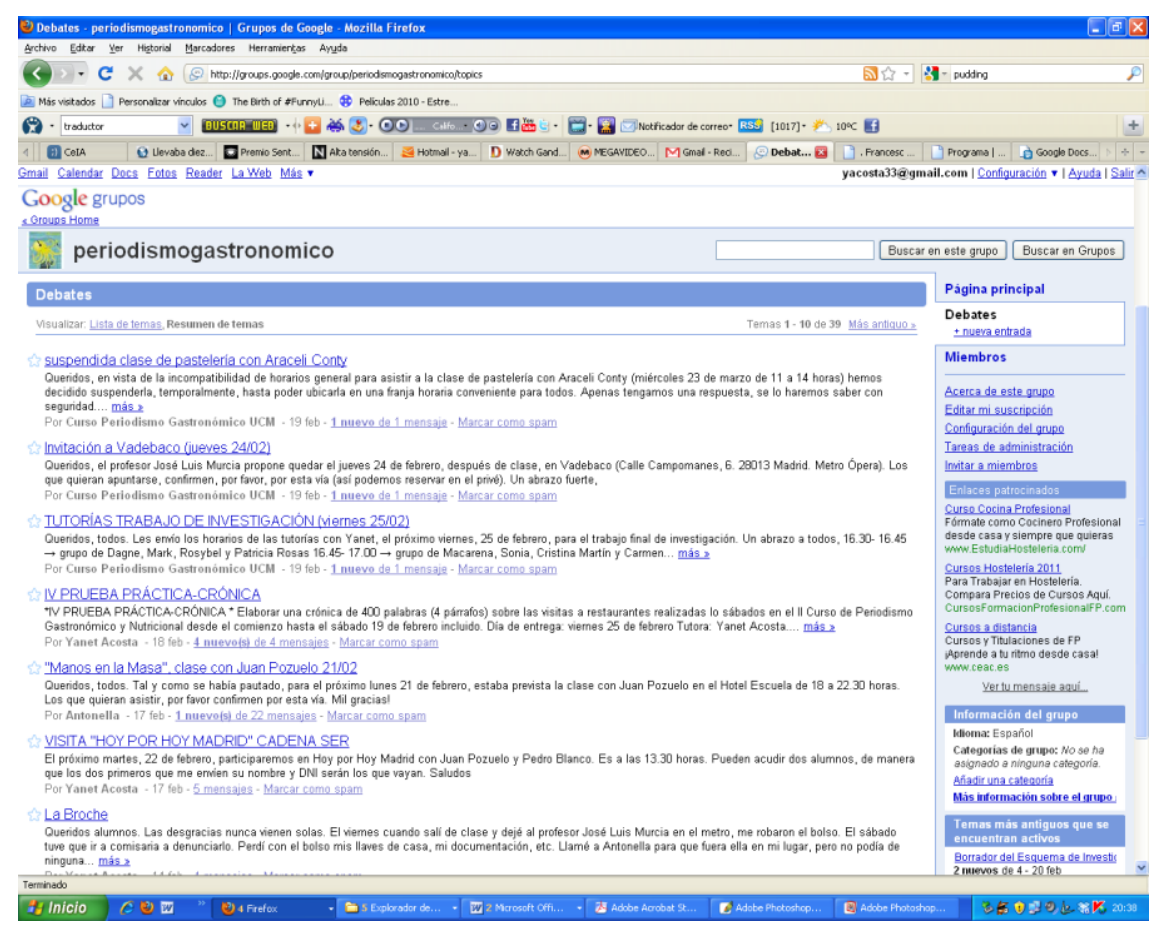

Figura 3: Imagen de Google groups

\subsubsection{Google docs}

Con esta herramienta de Google se pueden obtener listados en los que todos los usuarios, mediante el uso de un enlace, visualicen un documento en línea. Por ejemplo, pueden elaborar un listado de las mejores películas que explican un concepto impartido en clase o la bibliografía de la asignatura. Puede ser muy útil también a la hora de publicar el programa de la asignatura que se puede ir modificando a lo largo del curso. Es decir, se pueden enlazar documentos de sólo lectura o documentos susceptibles de ser editados, dependiendo del grado de seguridad que le imprima el creador del mismo. 


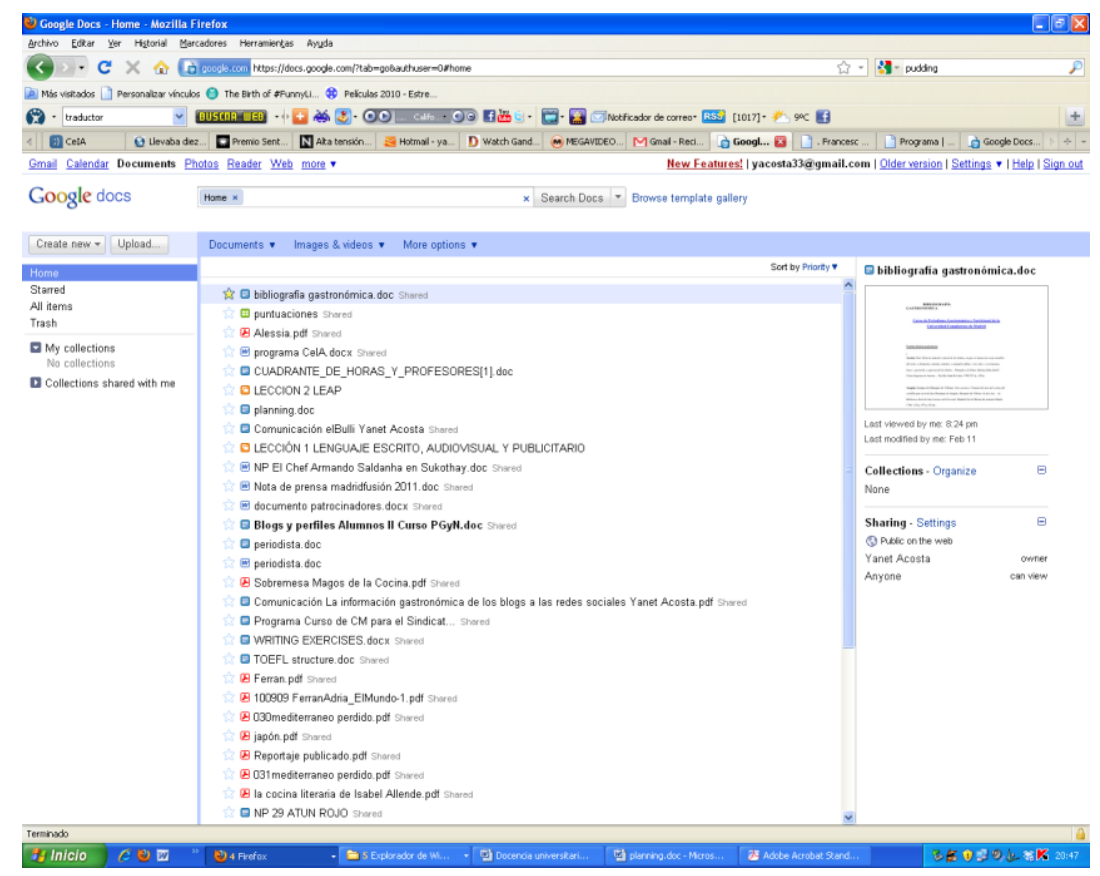

Figura 4: Imagen de Google docs

\subsection{Posterous}

Se trata de una red social en la que se pueden hacer grupos cerrados. Es interesante especialmente para aquellos alumnos que estudian cuestiones relacionadas con los medios audiovisuales. En ella, tan sólo a través de un mail, se pueden remitir fotografías que quedan fijadas en la plataforma como entradas de blog. De esta manera, cualquiera de los participantes puede hacer sus comentarios. Es especialmente útil para trabajos de clase en los que se solicite una serie de fotografías basadas en un tema, de forma que todos los alumnos pueden ver el trabajo del resto. También puede ser particularmente recomendable para grabaciones de audio o de imágenes. 


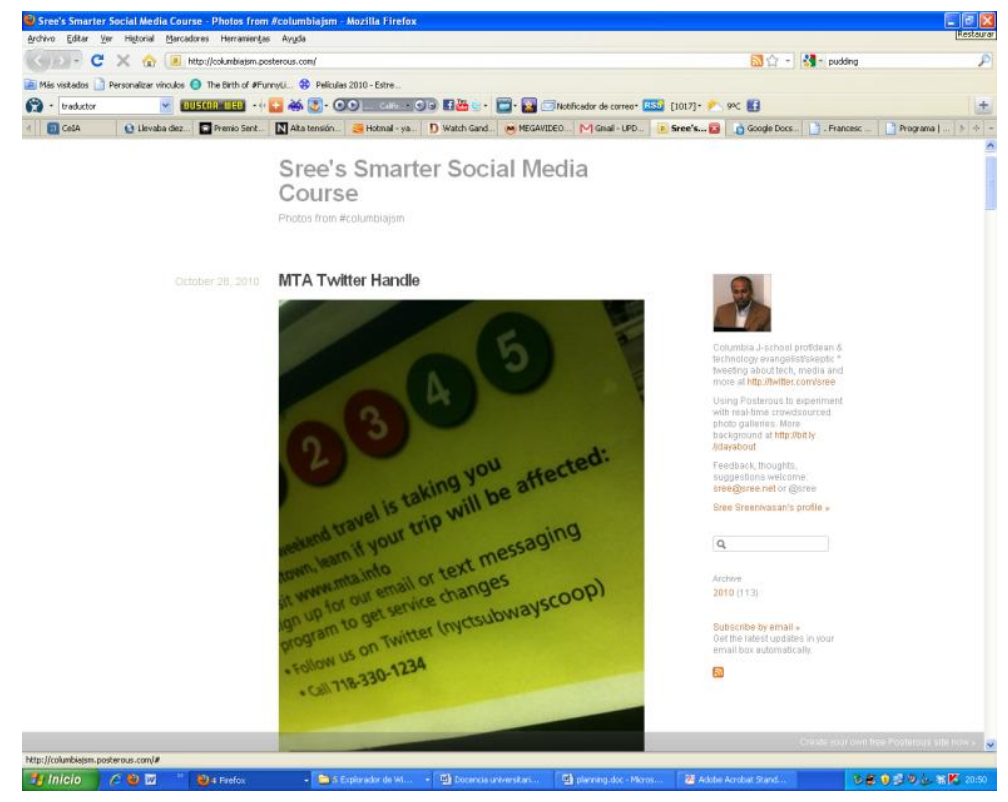

Figura 5: Imagen de Posterous

\subsection{Tuenti}

Tuenti es la red social por excelencia de los jóvenes en España. Unos 9,2 millones de usuarios activos en toda España (dato oficial de noviembre 2010), entre 14 y 24 años mayoritariamente, forman parte de esta red creada en 2006 por el estudiante estadounidense afincado en España, Zaryn Dentzel. Esta red permite a los profesores acercarse a los estudiantes a través de páginas en las que crear debates y proponer trabajos de sus asignaturas. Sin embargo, también conlleva un trabajo de convencimiento con los estudiantes para que asuman que el ocio y el estudio pueden ir de la mano. Estas páginas pueden ser cerradas o abiertas, en ellas, se pueden hacer comentarios o entradas de links a vídeos o textos relacionados con la asignatura.

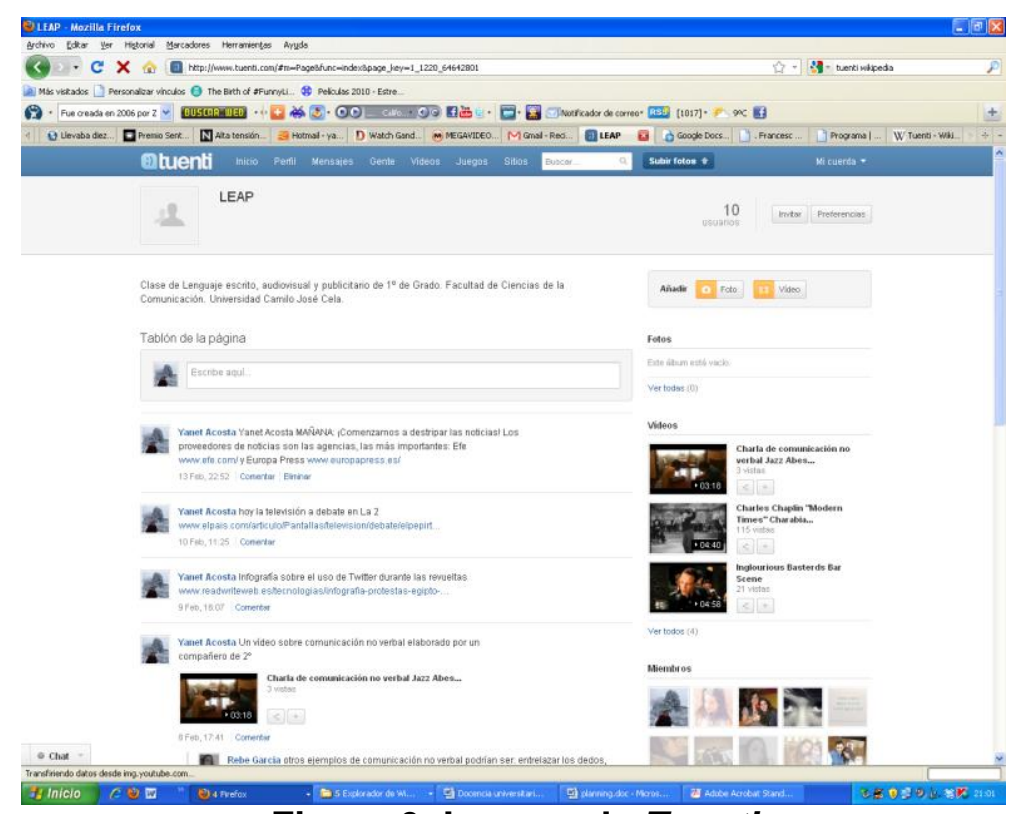

Figura 6: Imagen de Tuenti 


\subsection{Facebook}

Facebook es la red social más utilizada en España. Cuenta con unos doce millones de usuarios, habitualmente por encima de los 25 años. Esta red cuenta con diversas aplicaciones de gran interés para la formación como sus grupos. Éstos pueden ser abiertos o cerrados, según las personas seleccionadas. La usabilidad de los grupos se puede considerar superior a la de la red social Tuenti, ya que los debates se ordenan dependiendo de la última entrada que haya sido comentada y permiten subir documentos, algo que no es posible en Tuenti. Los grupos de Facebook pueden ser una herramienta ideal para los alumnos de posgrado o de los últimos años de carrera.

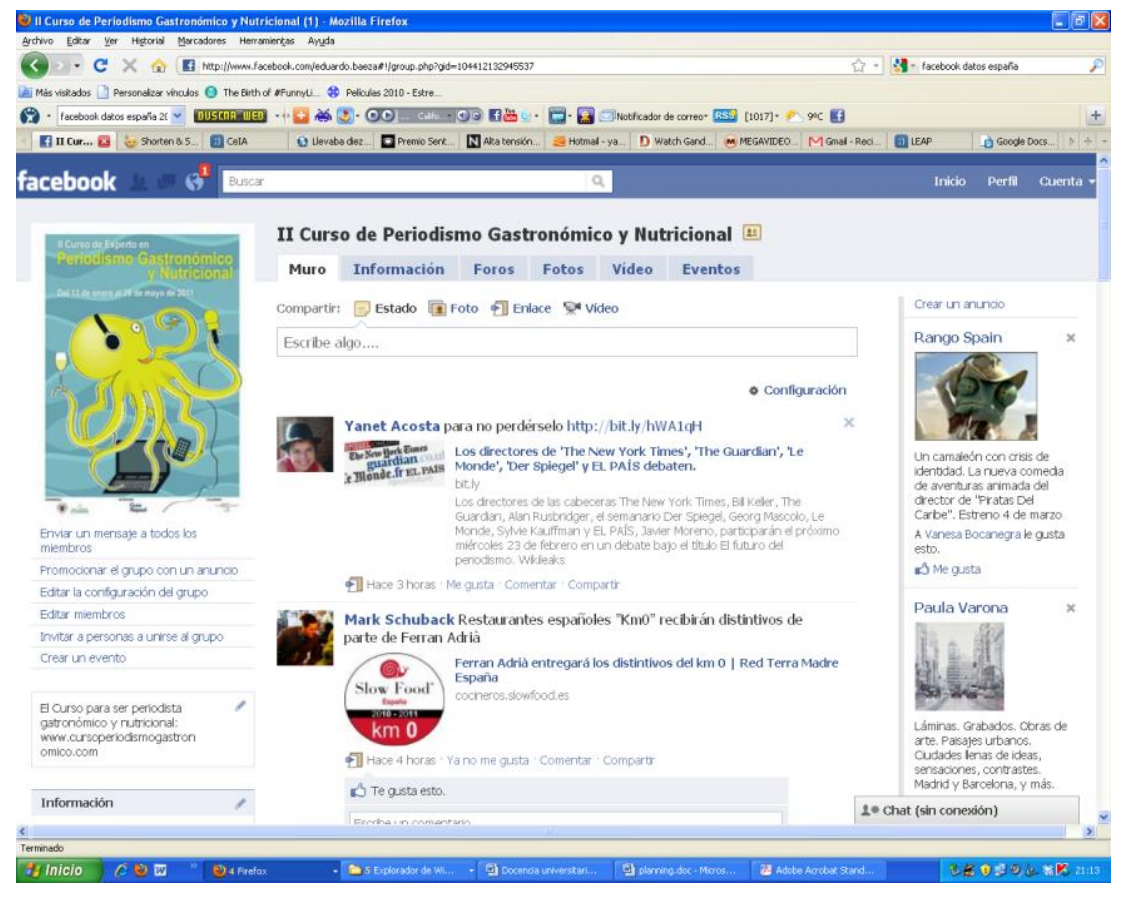

Figura 7: Imagen de Facebook

\subsection{Twitter}

Twitter es, hasta la fecha, la herramienta de microblogging por excelencia. A través de Twitter se pueden abrir debates, anunciar temas en clase o ideas propuestas para conocer la receptividad de las mismas no sólo entre los alumnos, sino también con los miembros que formen parte de esta red social. De esta manera, se convierte en una herramienta útil para la transferencia del conocimiento, eficaz por su instantaneidad al concebirse como una fórmula de comunicación en tiempo real.

Además, gracias al uso de Twitter los alumnos de las facultades de Comunicación, divulgan los contenidos que están creando en sus propios blogs o informan de lo que está sucediendo en ese momento en clase. De esta manera, se puede fomentar una mayor interactividad entre el alumnado y el profesorado, dependiendo de la asignatura que se imparta, a través de la creación de una etiqueta específica o hashtag que permitan recuperar los comentarios o mediante los tweets elaborados en torno a la materia. 


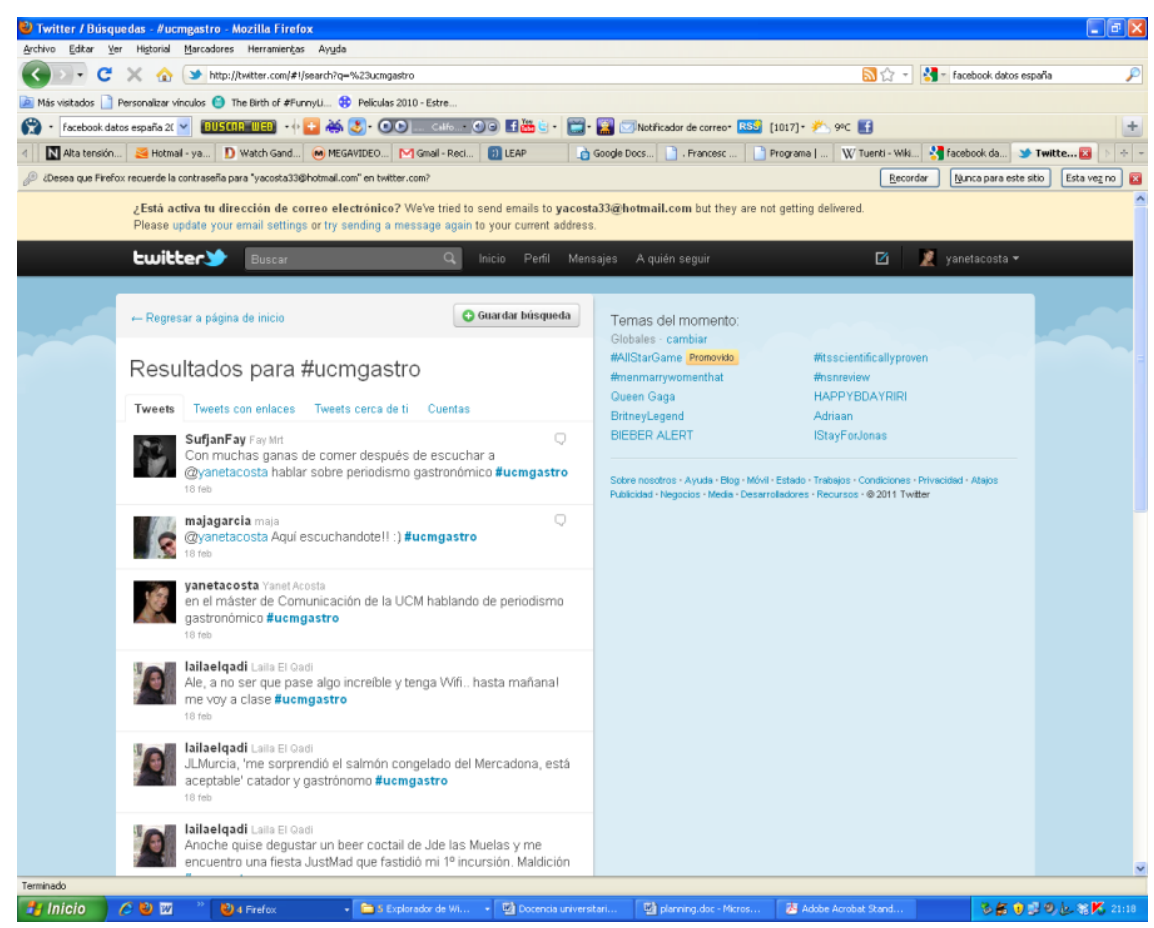

Figura 8: Imagen de Twitter

\subsection{Crear una red propia: Ning}

En ocasiones, los grupos de estudiantes quieren preservar su identidad, al igual que los profesores. En consecuencia, se puede crear una red propia en la que cada usuario se identifique con un objeto, una película o un símbolo, la red social Ning ha sido muy utilizada entre profesores de secundaria para fomentar esta interacción docente-discente. En cualquier caso, el riesgo de concebir Ning puede venir dado de la popularidad del resto de las redes sociales, por tanto, su uso puede ser inferior entre los estudiantes del grupo de clase, puesto que su preferencia esté destinada claramente hacia entornos donde se ubiquen sus conocidos y amigos como Facebook, Tuenti o Twitter.

Crear una red social únicamente con fines educativos puede conllevar intrínsecamente el riesgo de que, muchos de los alumnos, eviten su frecuencia a la hora de entrar al relacionarla directamente con la comunicación con el profesor de la asignatura, es decir, puede disminuir la motivación del estudiante en cuanto a su uso, tanto por estar habituado a otras redes más interactivas, como por asociarla particularmente a la universidad y al estudio.

\subsection{Rastrear el seguimiento de los alumnos: Bit.ly}

Bit.ly es una aplicación para acortar direcciones URL, con el que se puede contabilizar estadísticamente el número de veces que es cliqueado un enlace. La utilidad de esta herramienta reside en el conocimiento sobre cuántos alumnos han accedido a un contenido, que ha sido publicado en el campus virtual, si se envía el hipervínculo o enlace a la página a través de una dirección acortada con bit.ly. 


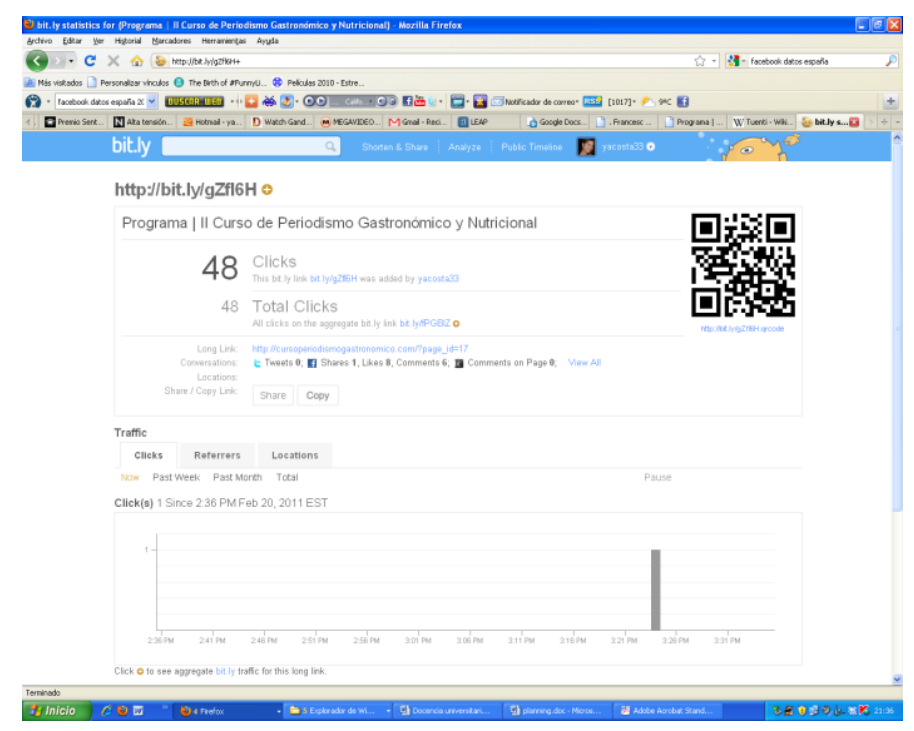

Figura 9: Imagen de bit.ly

\subsection{Encuestas online}

El feed-back de los alumnos es crucial para un profesor universitario. Las encuestas en papel sobre el profesor son habituales, pero también se pueden realizar de forma online con aplicaciones que ayudan a mejorar la gestión de las respuestas de los alumnos. Para ello se cuenta con EncuestaTick, una aplicación gratuita del portal de las encuestas.

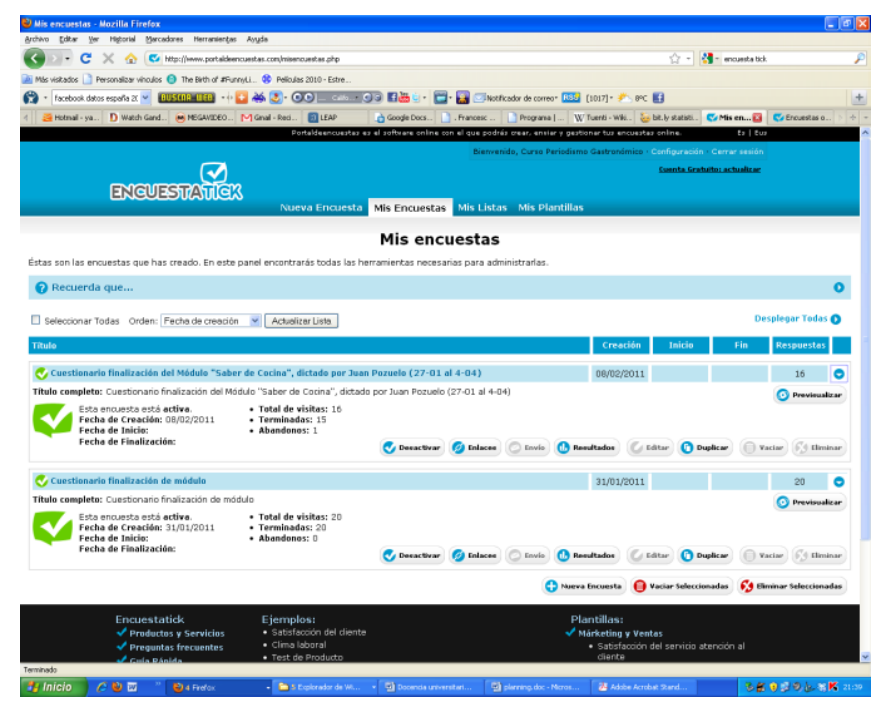

Figura 10: Imagen de EncuestaTick

3.10. Tutorías a través de aplicaciones de móvil: Whatsapp

La telefonía móvil ha incrementado exponencialmente en el uso de Internet, entre las aplicaciones que están teniendo una gran aceptación se encuentra el Messenger gratuito denominado 
Whatsapp, una herramienta de mensajería gratuita entre teléfonos móviles de una marca concreta. Estas conversaciones digitales pueden permitir el establecimiento de nuevas fórmulas de tutorías, llegando a superar a la telefonía en red con programas como Skype o a la comunicación vía mails.

\section{Conclusiones}

Después de todo lo explicado anteriormente, se pone de manifiesto la función tan destacada que pueden contraer las denominadas TIC en los procesos de aprendizaje y enseñanza en la formación universitaria. El desafío planteado de trasladar el aula universitaria hacia entornos virtuales y de colaboración puede constituirse como una realidad, de hecho, ya se están experimentando con estas nuevas aplicaciones como complemento a las clases magistrales, de manera presencial, con fines académicos. De alguna manera, el futuro de la docencia se perfila hacia esta combinación de docencia en el aula y formación mediante las nuevas tecnologías en línea, a modo de metodologías aplicadas a las TIC como el blended-learning.

La voluntad del profesor en adquirir estas nuevas destrezas, así como la de la involucrar a los propios alumnos, son claves a la hora de llevar a cabo estos nuevos mecanismos docentes. En este trabajo, se ha señalado al menos diez herramientas digitales, caracterizadas por la filosofía de colaboración web 2.0, en las que puede intervenir el profesor con sus respectivos alumnos. Entre ellas, se pueden resaltar la labor creativa e instructiva de redes sociales como Facebook, Ning o Twitter, así como las posibilidades brindadas por Google, por sistemas de encuentas online como Encuestas Tick y hasta por las nuevas fórmulas de mensajería virtual gratuita como Whatsapp.

\section{Bibliografía}

Buckingham, D. (2005): Educación en medios. Alfabetización, aprendizaje y cultura contemporánea, Barcelona, Paidós Comunicación.

Carr, N. (2011): ¿Qué está haciendo Internet con nuestras mentes? Superficiales, Madrid, Taurus. Castañeda, L. (2010): Aprendizaje con redes sociales. Tejidos educativos para los nuevos entornos, Sevilla, Eduforma.

De Haro, J. J. (2010): Redes sociales para la educación, Madrid, Anaya.

García, F. (2009): "Mito, ideología y realidad de un imaginario sociotécnico". Nativos digitales. Revista científica Icono 14, número 12.

Parra, D. y Álvarez, J. (2004): Ciberperiodismo, Madrid, Editorial Síntesis.

Pisani, F. y Piotet, D. (2008): La alquimia de las multitudes. Cómo la web está cambiando el mundo, Barcelona, Paidós Comunicación.

Piscitelli, A., Adaime, I. y Binder, I. (2010): El proyecto Facebook y la posuniversidad. Sistemas operativos sociales y entornos abiertos de aprendizaje, Barcelona, Editorial Ariel.

Redondo, J. A. (2010): Socialnets. La insospechada fuerza de unas aplicaciones que están cambiando nuestras vidas y nuestros trabajos, Barcelona, Ediciones Península.

Sierra, J. y Sotelo, J. (2010): Métodos de innovación docente aplicados a los estudios de Ciencias de la Comunicación, Madrid, editorial Fragua. 


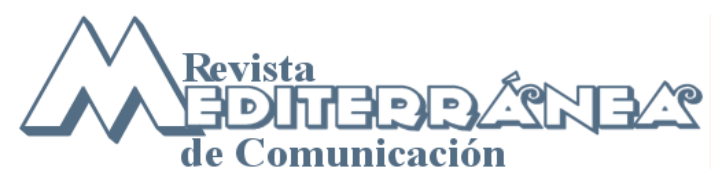

ISSN 1989-872X - Año2 (2011), pp. 43-58

Wolton, D. (2010): Informar no es comunicar. Contra la ideología tecnológica, Barcelona, Gedisa editorial. 